\title{
Crowdsensed Data Learning-Driven Prediction of Local Businesses Attractiveness in Smart Cities
}

\author{
Andrea Capponi $\sharp$, Piergiorgio Vitello $\sharp$, Claudio Fiandrino ${ }^{\star}$, Guido Cantelmo ${ }^{\dagger}$, \\ Dzmitry Kliazovich ${ }^{\star}$, Ulrich Sorger ${ }^{\sharp}$, Pascal Bouvry $\sharp$ \\ \# FSTC/CSC \& SnT, University of Luxembourg, Luxembourg, * IMDEA Networks Institute, Madrid, Spain, \\ $\dagger$ Technical University of Munich (TUM), Germany, ExaMotive, Luxembourg \\ E-mails: ${ }^{\sharp}$ \{firstname.lastname\}@uni.lu, `claudio.fiandrino@imdea.org, ${ }^{\dagger}$ g.cantelmo@tum.de, ${ }^{\star}$ kliazovich@iee.org
}

\begin{abstract}
Urban planning typically relies on experience-based solutions and traditional methodologies to face urbanization issues and investigate the complex dynamics of cities. Recently, novel data-driven approaches in urban computing have emerged for researchers and companies. They aim to address historical urbanization issues by exploiting sensing data gathered by mobile devices under the so-called mobile crowdsensing (MCS) paradigm. This work shows how to exploit sensing data to improve traditionally experience-based approaches for urban decisions. In particular, we apply widely known Machine Learning (ML) techniques to achieve highly accurate results in predicting categories of local businesses (LBs) (e.g., bars, restaurants), and their attractiveness in terms of classes of temporal demands (e.g., nightlife, business hours). The performance evaluation is conducted in Luxembourg city and the city of Munich with publicly available crowdsensed datasets. The results highlight that our approach does not only achieve high accuracy, but it also unveils important hidden features of the interaction of citizens and LBs.
\end{abstract}

\section{INTRODUCTION}

For decades, urban planners and researchers have relied on experience-based strategies and traditional methodologies to tackle urbanization issues. Nowadays, the pervasiveness of mobile devices permits to use Information and Communications Technology (ICT) by unleashing unprecedented possibilities in urban environments to improve citizens' quality of life [1] and services, such as public lighting [2]. Citizens carrying smart devices are a potential data source according to the mobile crowdsensing (MCS) paradigm [3], [4]. The crowd market is projected to raise from USD 385.1 Million of 2016 to USD 1142.5 Million by 2021 at a compound annual growth rate of $24.3^{1}$. Incentives and energy-efficient schemes are typically employed to make MCS systems effective [5].

LBs require their owners (e.g., companies, individuals, and institutions) to take decisions for profit maximization and to offer competitive services to customers. The most crucial decisions include the typology of a LB and its location when opening, but also setting prices, the number of required employees per hour, and opening hours for proper management. Effective strategies to boost LBs require knowledge of the complex dynamics of urban environments, which depend on the spatial distribution of citizens and locations [6]. For instance, understanding real-time citizens' mobility as well as forecasting significant flows of citizens moving to a specific urban area for a special event help municipalities to manage crowds and entrepreneurs in deciding suitable locations and required staff. To develop solutions for smart mobility [7], testbeds

${ }^{1}$ https://www.marketsandmarkets.com/PressReleases/crowd-analytics.asp are prohibitively costly and relying on simulators to model pedestrian trajectories and crowdsensing activities [8] is a valid cost-effective alternative.

Traditional approaches to investigate LBs popularity rely either on surveys that capture users' preferences or cellular traces that infer urban mobility [9]. However, such approaches are prone to users misbehavior, technical limitations (e.g., poor network coverage), and datasets available only from network operators [10]. Crowdsensed data-driven approaches may provide novel solutions in this direction by exploiting MCS systems and services like Google Maps that make available accurate information on travel times and popularity of LBs.

This work aims to bring one step further the research on urban computing and to boost LBs popularity by overcoming the limitations of historically experience-driven approaches. We leverage crowdsensed data to enforce highly-accurate classification of LBs category and attractiveness with ML techniques that are powerful to handle massive data volumes and widely employed from a variety of applications, such as to infer and predict human mobility in urban context [11]. In this work, we show that typical urban metrics (e.g., the centrality of places in street networks) fail to properly classify LBs, while combining crowdsensed data (e.g., peak hours in LBs) with basic ML techniques supports and improves typical experience-driven approaches. To illustrate with few representative examples, restaurants and pubs usually concentrate in close areas and influence one with each other, while LBs like pharmacies are uniformly distributed over a city. Also, reachability by public transport significantly impacts on LBs popularity.

\section{BACKGROUND}

\section{A. Related works}

Understanding urban mobility and travel behavior is fundamental to investigate the popularity of LBs. Typical approaches infer mobility employing cellular traces. In [12], the authors show the evolution of citizens movements during the day in Milan, Italy. Statistical inference and cluster analysis applied to cellular traces can forecast the influence of special events on the daily mobility and activities of the users [13]. Other studies focus on factors that influence citizens mobility, activities and district attractiveness, such as cultural events [14]. Unfortunately, these approaches present significant shortcomings due to the misbehavior of users, lack of location accuracy and technical limitations, such as poor network coverage and unavailability of datasets obtained from network operators [10]. 
Crowdsensed data-driven approaches can be applied to better tackle urbanization issues and understand citizens mobility [15]. In this context, MCS permits to directly gather data from users and infer their mobility patterns with high accuracy, e.g., classify residents and visitors and identify special events [16].

By feeding ML techniques with crowdsensed data can open up unexplored solutions. ML approaches are extensively used to extract information from large datasets acquired from various sources [17]. ML and deep learning models were applied to various areas, such as speech or image recognition, and in urban scenarios to detect accident risks [18] and predict traffic flows [19]. The use of learning techniques over crowdsensed data has become a win-win solution in different domains, such as to predict human judgments of pairwise image comparisons and produce urban perception at large scale [20]. Unlike the above proposals, this work aims to provide predictions of the popularity of LBs inferred by studying human mobility in urban environments. The closest work to our is [21] that has the objective of predicting the temporal dynamics of newly established LBs by exploiting the check-in data of Foursquare users. By contrast, we study generic LBs and rely on Google Popular Times data.

\section{B. Crowdsensed datasets}

Mobile users have at disposal several ways to share data such as location-based social networks (LBSN) (e.g., Facebook, Foursquare, Twitter, etc.), and crowdsourced applications (e.g., OpenStreetMap, Waze). Such contribution has made available large datasets that enable an analysis of citizens mobility, travel behaviors, accessibility of urban areas and popularity of LBs. In particular, Google makes available anonymized crowdsensed data passively collected from Google Maps users who opted into Google Location History ${ }^{2}$. These datasets include information on customers visits to LBs, such as popular times per hour, the average duration of visits, queuing time to access a service, and real-time popularity.

In this work, we exploit Google Popular Times with a twofold purpose. First, we investigate features that can influence the popularity of LBs. Second, we feed ML techniques on such dataset to classify category and attractiveness of LBs according to the considered features.

\section{PREliminary ANALysis}

This Section grounds the roots of our work by showing why traditional urban metrics fail to classify and predict LBs attractiveness properly.

Weekly popularity: Google Popular Times define the temporal profile of a LB as a vector of normalized per-hour weekly values in the range $[0: 100]$. In particular, 0 defines closing hours, 1 the lowest amount of visits per-hour in a week, and 100 the highest. The use of normalized values permits to analyze the trend of LBs during a week and its influencing factors (e.g., LBs that have more success at weekends in touristic areas or at lunchtime in business districts). This hides the degree

\footnotetext{
${ }^{2} \mathrm{https} / / /$ support.google.com/business/answer/2721884
}

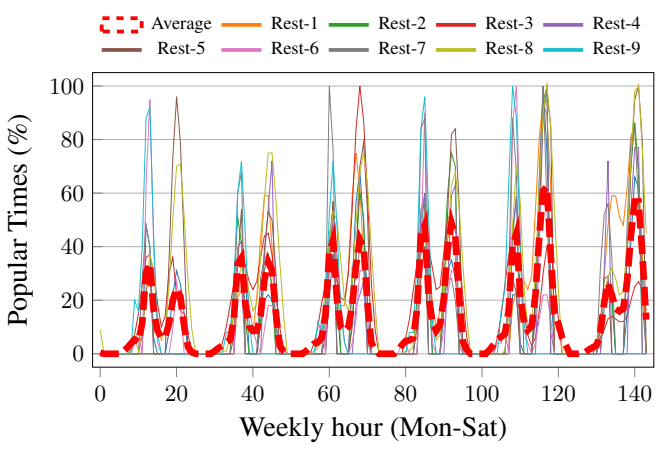

(a) Average popularity of restaurants in Ville Haute - Gasperich • Limpertsberg • Kirchberg • Gare • Outlier

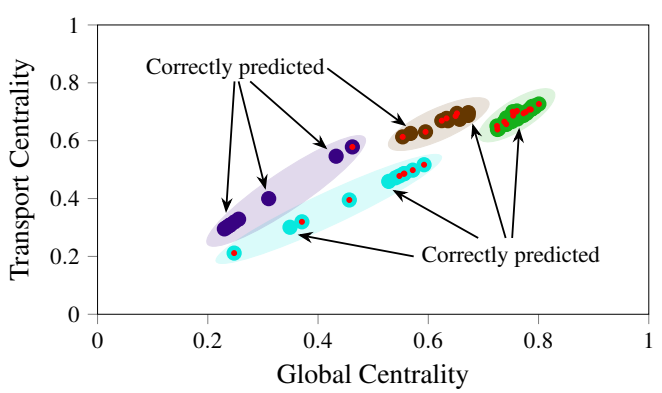

(b) Centrality and similarity

Fig. 1. Data aggregated from different Luxembourg districts for restaurants of success of a single LB (e.g., having more customers than others), which is however not the purpose of this work.

Fig. 1(a) presents Popular Times of nine restaurants and their average from Monday to Saturday in Luxembourg city (Ville Haute), a district with offices (banks, public institutions), shops, tourists spots and places for nightlife (bars, pubs). Sunday is excluded because no information was available in the dataset. The peaks of popularity approximately at $12,20,36$, 44, etc., correspond to lunch (12 PM) and dinner (8 PM) times of each day. Analyzing the peaks in pairs, we can compare the trend of restaurants day by day and understand the lifestyle of the district. During weekdays the peaks are around lunch time or equally spread at lunch-dinner time (restaurants full of workers) while on Saturday at dinner time because most offices are closed. Friday is the most popular day at both lunch and dinner times because both workers, tourists, and citizens populate restaurants.

Centrality and similarity: The popularity of LBs depends on their proximity with other LBs and accessibility through public transportation. The centrality metric defines the importance of individual nodes in a network and can quantify popularity Specifically, we consider the closeness centrality, defined as the sum of the length of the shortest paths between a node and all other nodes within the street network. We measure global-centrality and transport-centrality. The global-centrality defines the proximity of a LB with all other LBs:

$$
C_{B}(k)=\frac{N_{B}-1}{\sum_{i \neq k} d_{k i}},
$$

where $k$ is the $k$-th node, $N_{B}$ is the total number of LBs and $d_{k i}$ is the distance between a couple of nodes. The transportcentrality measures the proximity of a LB with respect to 
transport facilities:

$$
C_{T}(k)=\frac{N_{T}}{\sum_{i \neq k} d_{k i}},
$$

where $N_{T}$ is the total number of transportation access points (e.g., bus stops or metro stations) and $d_{k i}$ is the distance between the considered LB and a transport node. Considering the Earth as an oblate ellipsoid, the distance is computed with the shortest geodesic path [22]. While popularity measured with centrality identifies time-invariant characteristics of a LB, the similarity compares two LBs temporal profiles. The similarity aims to correlate LB weekly popularity to the average of all LBs in the same district. To measure similarity, we exploit the symmetric index of Jensen-Shannon divergence (JSD) that outperforms the asymmetric Kullback-Leibler divergence (KLD) [21]. The similarity of two LBs $i$ and $j$ is:

$$
J\left(D_{i}, D_{j}\right)=H\left(\frac{D_{i}+D_{j}}{2}\right)-\frac{H\left(D_{i}\right)+H\left(D_{j}\right)}{2},
$$

where $H$ is the Shannon entropy, $D$ is the temporal profile of a LB, and $J$ represents the divergence of two temporal profiles, The similarity can assume values in the range $[0-1]$. 0 represents the maximum similarity (e.g., the temporal pattern of a shop with itself) and 1 represents the maximum divergence.

Fig. 1(b) links centrality and similarity metrics in Luxembourg city. The clusters represent four districts, in line with global- and transport-centrality of restaurants. Red dots represent LBs whose weekly temporal demand is closer to the average of other districts (outliers). On the contrary, dots of the same dominant color have a weekly pattern more similar to their geographical district. With the sole exception of Kirchberg district, most of the LBs are marked as outliers. Therefore, this analysis unveils that centrality and similarity are not enough to assess the popularity of LBs and their relationship with districts. The analysis correctly predicts the popularity of LBs in Kirchberg because the district is geographically separate from other districts of the city and it is home of European agencies, insurance and financial companies making the LBs in the area to share peculiar popularity trends. This paper shows how to overcome this shortcoming by enforcing a ML-based analysis of the same dataset.

\section{ML-Augmented Methodology}

This Section describes the methodology for applying ML techniques to crowdsensed data. The Popular Times datasets undergo a procedure to extract features and determine the most suitable inputs to train the ML algorithms. We select only the features that augment the output accuracy after the training phase, while the others are discarded. For space reasons, we omit this preliminary selection. Next ( $\mathrm{IV}-\mathrm{A})$, we introduce the ML algorithms. Then ( $\S$ IV-B), we discuss the considered multi-classification problems, extracted input features, and output classes. Each output is classified by exploiting a onevs-all approach. For each LB, the element corresponding to the predicted class is set to one, all others to zero.

\section{A. Machine Learning Techniques}

This study considers Support Vector Machine (SVM) with a Gaussian kernel and MultiLayer Perceptron (MLP) neural network techniques for multi-classification problems. The choice is due to characteristics of our study, which presents a small number of features $N$ (e.g., $1-1000$ ), and an intermediate number of $M$ training samples (e.g., $1-50000$ ). The chosen ML approaches perfectly fit this scenario. Similar ML techniques like logistic regression or SVM without kernel (or linear kernel) have not been considered because they perform better when $N$ is relatively large if compared to $M$ (e.g., 10000 and $M$ between 1 and 1000). In the following, we briefly analyze the considered ML techniques.

Support Vector Machines (SVMs) aim to classify input samples into output classes by dividing a hyperplane with an optimal boundary through kernel methods. To this end, it is crucial to perform fine tuning of the regularization parameter, typically named $C$. Furthermore, employing a kernel based on a gaussian function, it is required to set the standard deviation, indicated as $\gamma . C$ trade-offs the correct classification of training samples and the smooth decision boundary. Small values lead to simple decision functions, which correspond to a higher tolerance to errors and smooth the classification on the training dataset. On the contrary, high values correspond to a classification with minimal error and a hyperplane with a small margin. Intuitively, $\gamma$ defines how a single training sample influences other points according to its distance from the boundary.

Multilayer Perceptron $(M L P)$ is a feedforward artificial neural network that takes a vector as input and maps it into another vector as output. It is based on different hidden layers that connect inputs to outputs. Each layer includes a certain number of nodes and nodes of different layers are connected by links with different weights. The output of a node at each layer is given by the weighted sum of all inputs. Each node in the hidden layers is connected to all nodes of next and previous layers for a fully connected topology.

\section{B. Predicting LBs Category and Attractiveness}

We formulate two multi-classification problems to predict LBs category and attractiveness by feeding ML techniques with input features extracted from crowdsensed data.

Extracted features: After a preliminary analysis that we omit for space reasons, we select as input features from the large available datasets those that performed better and we categorize them as intrinsic and extrinsic. Intrinsic features are given by geo-location characteristics and owners' decisions, which do not present a high variability over time (e.g., opening hours and type of service offered). These properties are already widely exploited in traditional approaches for urban analytics. Extrinsic features depend on the temporal interactions of citizens with LBs, such as waiting time and average time of staying. They change more rapidly than the intrinsic ones and depend on several factors, e.g., special events, time of day, day of the week, etc. The intrinsic features we consider are global-centrality, transport-centrality, opening hours, and category. The parameters that define centrality have already been discussed in Sec. III. Opening hours consists of an array of 144 binary values (Mon-Sat) that shows when a LB opens. For 
each hour in a weekday, the value 0 indicates closing time and 1 opening time. The category depends on the service offered by LBs. The extrinsic features are popular times, average time of visit, and average waiting time. Popular times were discussed in Sec. III. The average remaining time defines in minutes the duration of customers' visits. The average waiting time indicates the minutes while waiting to access the service.

Output classes: LB categories depend on the service offered by a LB. Output classes are public, store, health, restaurant, and bar. The class public indicates generic services and offices for the community, such as institutions, post, financial and insurance companies. Store includes each kind of shop or seller for any goods, such as supermarkets, clothing, bakeries, etc. Health comprises public and private places related to healthcare, e.g., hospitals, medical centers, dentists, and specialists. Restaurant includes all LBs that prepare meals with seating places. Bar consists of LBs selling mainly drinks, but can also include meals, e.g., pubs.

LB attractiveness is classified into working, nightlife, weekend, business hours (Bus. H.), and shopping hours (Shop. H.) Working indicates LBs with peak hours during break times of working areas, such as weekdays at mid-morning and lunchtime. It comprises typically shopping malls, bars, fast foods and some types of restaurants. The class nightlife shows peak hours at dinner times during all week and overall on weekends, including restaurants, pubs, and clubs. Weekend describes low popularity on weekdays and peak hours at weekends, which is typical of shopping malls located far from working areas and touristic places. Business hours indicates typical opening times and consequent popular hours of public offices, from early morning to mid-afternoon including lunch breaks. Shopping hours include the typical popularity hours of shops for different goods, presenting a uniform distribution on both weekdays and weekends during daytime.

\section{DATA-DRIVEn Evaluation}

This Section first presents simulation set-up and performance metrics, then the obtained results.

\section{A. Setting}

To conduct the evaluation, we employ publicly available Popular Times of LBs for Luxembourg city and the city of Munich downloaded between July 21st and July 30th, 2018. These two cities present different characteristics in terms of morphology, size, street topology, and lifestyles of residents and visitors. This permits to conduct an effective analysis and discussion of the obtained performance. The datasets include 1084 and 3784 LBs for Luxembourg city and Munich respectively and are proportionally divided in $80 \%, 10 \%$, and $10 \%$ for training, cross-validation, and test phases respectively. The performance evaluation exploits Scikit-learn, which is a Python-based open-source library.

To predict the LBs category, the input features are: average opening hours, time spent, global-, and transport-centrality. In this case, we restrict the datasets to the LBs for which information on time spent is available (800 and $1600 \mathrm{LBs}$ for
Luxembourg city and Munich respectively). The hyperparameters in the SVM approach are set to $C=2^{8}$ and $\gamma=2^{-12}$ We will further discuss the rationale about the selection of parameters (see discussion Fig. 3a). In the MLP approach, an exhaustive search with a grid-search algorithm leads to the choice of one hidden layer with 13 nodes.

For LBs attractiveness, the considered features are opening hours, category, district, popular times, global-, and transportcentrality. In this case, the entire datasets were employed. The methodology followed to set the hyperparameters is as for the LBs category. For SVM, the parameters are $C=2^{6}$ and $\gamma=2^{-10}$ (likewise above, the rational is discussed in Fig. 3b), MLP consists of 8 nodes per layer with 2 hidden layers.

\section{B. Performance Metrics}

We consider precision, recall, F1 score, and accuracy indexes. While precision, recall, and F1 score are per-class measures, the accuracy averages the measures of all the classes. For completeness of the analysis, we consider i) true positive $(t p)$ and true negative ( $t n$ ) values to indicate respectively a correct prediction of positive or negative class; (ii) false positive ( $f p$ ) and false negative ( $f n$ ) values to denote an incorrect prediction. In this context, a positive observation indicates the class under analysis, while a negative observation indicates all the other classes, according to the one-vs-all approach.

The precision indicates the ratio of correct positive predictions over the total predicted positive occurrences $(t p /(t p+f p))$. In other words, it indicates the capacity of the model to not predict another true class as the actual class. The recall is the ratio of correct predictions on positive observations to all the occurrences in class under analysis $(t p /(t p+f n))$. It indicates the capability of the model to catch all the samples of a class. The F1 score is the weighted average of precision and recall indexes and analyzes incorrect predictions. Typically, the F1 score is very useful to unveil insights from results when false positives and false negatives have different costs. The accuracy is computed as the ratio of correct predictions over the total occurrences and defines the performance of a classifier. Specifically, accuracy is the optimal performance indicator when the classes are symmetric, i.e., incorrect predictions have the same weights.

\section{Results}

Table I presents detailed results on precision, recall, F1 score, and accuracy for the predicted categories in both cities with MLP and SVM approaches. The prediction on LB categories presents higher accuracy for Luxembourg city with both ML techniques. Viceversa, Munich shows higher accuracy in predicting LBs attractiveness. Regarding the ML techniques, SVM presents an overall accuracy higher than MLP. The Table clearly shows that precision achieves high values for categories of restaurant, health, and store, while it is low for bar and public because these categories share common characteristics with other categories. The LBs prediction precision varies in the two cities because it depends on specific characteristics of each city, mainly type of visitors (e.g., tourists, workers, or residents) and city lifestyle (e.g., commercial, touristic, 
TABLE I

STATISTICS FOR LB CATEGORY AND ATTRACTIVENESS PREDICTION

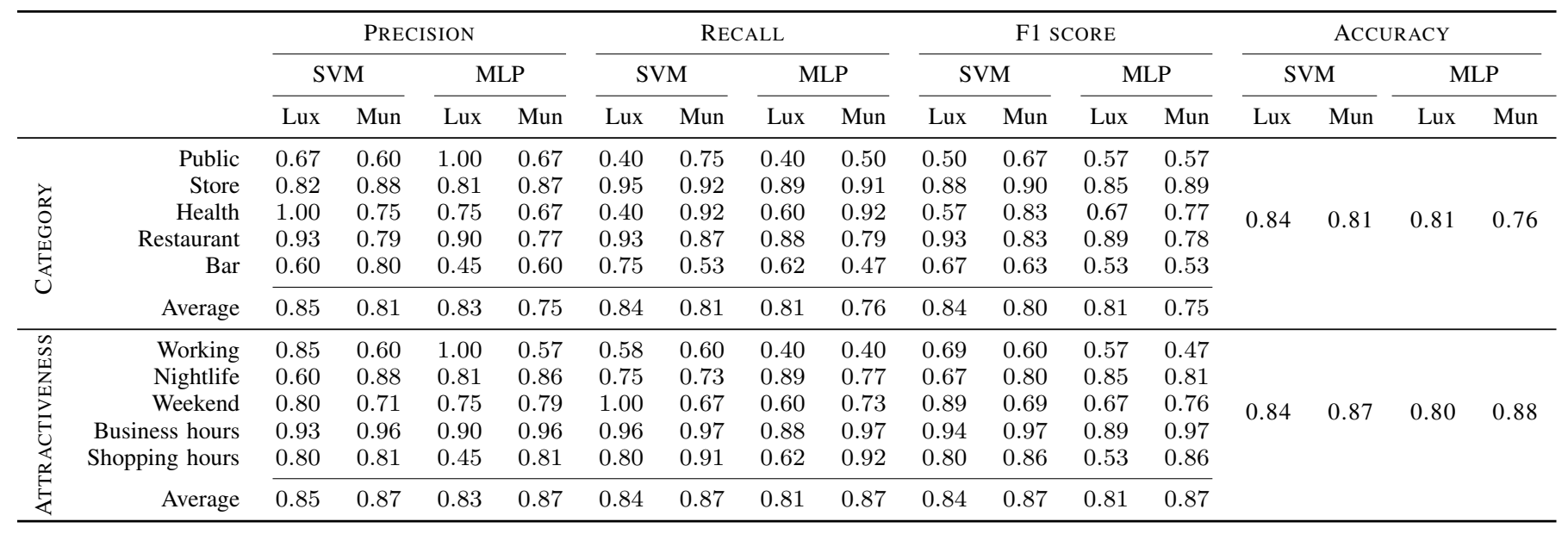

or working areas). For example, restaurants present higher values of precision in Luxembourg city because the opening hours are not as international as in a larger city like Munich, while bars are predicted with higher precision in Munich. In Luxembourg city, bars and restaurants share opening hours while in Munich pubs and clubs open until late night, unlike restaurants. Regarding the attractiveness, on the one hand, the class working is predicted with much higher precision in Luxembourg. The reason is as follows: LBs with peak visits during job breaks are typically not popular, i.e., receive lower visits during other moments of the day or with another type of customers (e.g., tourists at the weekend). On the other hand, the class working is not well predicted in Munich because LBs are popular at different times during the day with no distinctive working areas. Business and shopping hours present higher values in Munich because of its urban plan characterized by LBs concentrated in specific districts with easily recognizable peak hours (e.g., the city center and shopping malls). For similar reasons, note that the model catches most samples of class (recall index) for restaurants and stores with both cities and both techniques when predicting the category, and business and shopping hours when predicting the attractiveness. F1 score analyzes the incorrect predictions by presenting a weighted average of recall and precision and the results are in line with previous considerations.

To simplify understanding, Fig. 2 depicts confusion matrices to highlight single occurrences for each true and predicted class and summarizes the prediction results. Each cell contains a value that indicates the number of occurrences of a predicted class when testing true inputs. The colors in legend bars represent the percentage of correct predicted occurrences over the total of true class values, which corresponds to the recall index between 0 and 1 . The columns show predicted class values. The sum of all values in each row indicates the total occurrences for such class. The occurrences of correct predictions for each class are in the diagonal. The accuracy is the sum of all elements on the diagonal on all elements of the matrix. The analysis on the confusion matrices allows to i) discuss and compare behaviors of different LBs and ii) extend the discussion in point i) to different cities. As expected and already pointed out in Table I, categories with distinctive features present a better prediction. The results in the table, however, do not show the wrong occurrences as confusion matrices allow. The categories restaurant and store achieve higher recall for both ML techniques in both cities because LBs in these categories share distinctive characteristics like opening hours. On the opposite, public and bar have a lower recall, and their wrong predictions occur respectively in store and restaurant. These LBs offer services with similar daily patterns, e.g., stores - public offices, and bars - restaurants. Fig. 2(a) and Fig. 2(b) clearly highlight these considerations because in Luxembourg city 2 bars over 8 are predicted as restaurants whereas for Munich this occurs for 14 LBs over 34 . Note that the health category achieves significantly different results in the two cities. The motivation is the different number of LBs in the available datasets. In this case, higher precision is attributed to a larger dataset.

When analyzing the attractiveness, Fig. 2(c) and Fig. 2(d) unveil that the highest number of prediction errors occur for working and nightlife classes. As previously discussed, the motivation is that restaurants and bars exhibit a high popularity at lunch and dinner times, which are typical characteristics shared between working and nightlife classes. For instance, Fig. 2(c) and Fig. 2(d) respectively show that in 4 occurrences over 15 and in 4 over 16 working class true values are predicted as nightlife. The highest number of correct predictions occur for business hours ( 51 over 53 in Luxembourg city, 156 over 160 in Munich), as the popularity is uniform during all weekdays. By comparing the two cities, Fig. 2(c) and Fig. 2(d) show that it is easier to predict the weekend class in Luxembourg city (4 over 4) than in Munich (10 over 15). While Luxembourg city is a destination popular for business and not for tourism, the amount of visits in LBs varies consistently between weekdays and weekends. On the opposite, in Munich it varies only a little.

Fig. 3 shows a study on the dataset of Munich for choosing 


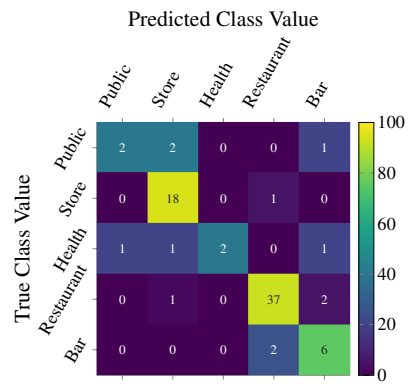

(a) Category - Lux

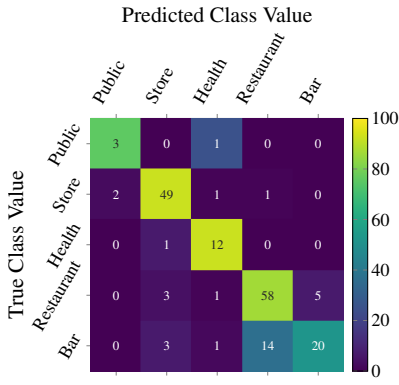

(b) Category - Munich

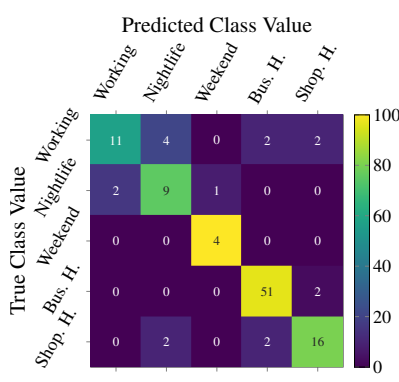

(c) Attractiveness - Lux

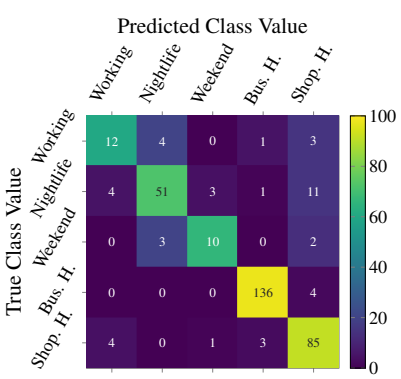

(d) Attractiveness - Munich

Fig. 2. Confusion matrices for LBs category and attractiveness prediction with SVM technique. The rows show true class values and the columns show predicted class values.

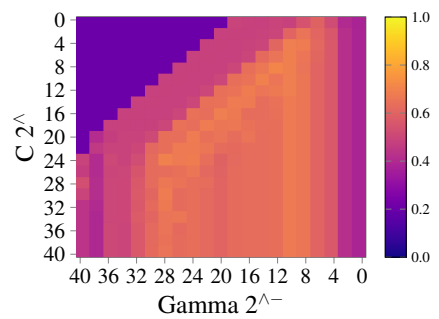

(a) Category

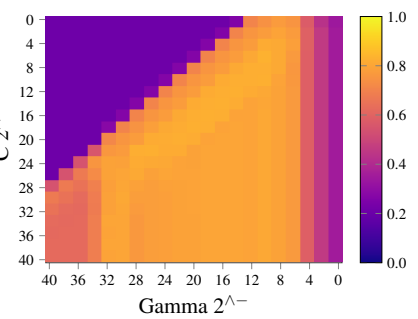

(b) Attractiveness

Fig. 3. Analysis of F1 score to optimize the SVM parameter selection for Munich. The values range between 0 and 1 .

the best parameters fitting the SVM technique in predicting LB category and attractiveness. Results are obtained by considering the F1 score to seek a balance between Precision and Recall. Specifically, SVM optimization parameters are $C=2^{8}$ and $\gamma=2^{-12}$ for LB category prediction, while they are $C=2^{6}$ and $\gamma=2^{-10}$ for LB attractiveness prediction.

\section{CONCLUSION}

This paper applies ML techniques on crowdsensed data from citizens to perform accurate predictions of LB category and attractiveness. Specifically, the work relies on Google Popular Times datasets and shows that ML-driven analysis outperforms historical urban computing metrics. After a preliminary analysis, the LB category and attractiveness are predicted using two different subsets of features extracted from crowdsensed data. The conducted evaluation shows that data-driven approaches outperform traditional urban metrics. The results unveil that classes exhibiting similar behaviors present higher errors when predicting their occurrences. For instance, the attractiveness of nightlife and working in a large-scale city like Munich can be miscategorized because they both include many restaurants and bars.

\section{REFERENCES}

[1] R. Ranjan, M. Wang, C. Perera et al., "City data fusion: Sensor data fusion in the Internet of Things," Int. J. Dist. Sys. Tech., vol. 7, no. 1, pp. 15-36, Jan 2016.

[2] G. Cacciatore, C. Fiandrino, D. Kliazovich et al., "Cost analysis of smart lighting solutions for smart cities," in Proc. IEEE ICC, May 2017, pp $1-6$.

[3] A. Capponi, C. Fiandrino, B. Kantarci et al., "A survey on mobile crowdsensing systems: Challenges, solutions and opportunities," IEEE Communications Surveys Tutorials, pp. 1-49, May 2019.

[4] A. Capponi, C. Fiandrino, D. Kliazovich et al., "A cost-effective distributed framework for data collection in cloud-based mobile crowd sensing architectures," IEEE Trans. on Sustainable Computing, vol. 2, no. 1, pp. 3-16, Jan 2017.

[5] M. Tomasoni, A. Capponi, C. Fiandrino et al., "Why energy matters? profiling energy consumption of mobile crowdsensing data collection frameworks," Pervasive and Mobile Computing, vol. 51, pp. 193 - 208, 2018.

[6] Y. Zheng, L. Capra, O. Wolfson et al., "Urban computing: concepts, methodologies, and applications," ACM Trans. on Intelligent Systems and Technology (TIST), vol. 5, no. 3, p. 38, 2014.

[7] A. Bucchiarone and A. Cicchetti, "A model-driven solution to support smart mobility planning," in Proc. of ACM/IEEE MODELS, 2018, pp. $123-132$.

[8] C. Fiandrino, A. Capponi, G. Cacciatore et al., "CrowdSenSim: a simulation platform for mobile crowdsensing in realistic urban environments," IEEE Access, vol. 5, pp. 3490-3503, Feb 2017.

[9] F. Calabrese, M. Diao, G. Di Lorenzo et al., "Understanding individual mobility patterns from urban sensing data: A mobile phone trace example," Transportation research part C: emerging technologies, vol. 26, pp. 301313, 2013.

[10] X. Wang, Z. Zhou, Z. Yang et al., "Spatio-temporal analysis and prediction of cellular traffic in metropolis," in IEEE ICNP, Oct 2017, pp. $1-10$.

[11] Y. Zhou, B. P. L. Lau, C. Yuen et al., "Understanding urban human mobility through crowdsensed data," IEEE Communications Magazine, vol. 56, no. 11 , pp. 52-59, Nov 2018.

[12] C. Ratti, D. Frenchman, R. M. Pulselli et al., "Mobile landscapes: Using location data from cell phones for urban analysis," Environment and Planning B: Planning and Design, vol. 33, no. 5, pp. 727-748, 2006.

[13] F. Calabrese, M. Colonna, P. Lovisolo et al., "Real-time urban monitoring using cell phones: A case study in rome," IEEE Trans. on Intelligent Transportation Systems, vol. 12, no. 1, pp. 141-151, Mar 2011.

[14] D. Hristova, L. M. Aiello, and D. Quercia, "The new urban success: How culture pays," Frontiers in Physics, vol. 6, p. 27, 2018.

[15] C. Chen, J. Ma, Y. Susilo et al., "The promises of big data and small data for travel behavior (aka human mobility) analysis," Transportation research part C: emerging technologies, vol. 68, pp. 285-299, 2016.

[16] M. S. Kaiser, K. T. Lwin, M. Mahmud et al., "Advances in crowd analysis for urban applications through urban event detection," IEEE Trans. on Intelligent Transportation Systems, pp. 1-21, 2017.

[17] C. Zhang, P. Patras, and H. Haddadi, "Deep learning in mobile and wireless networking: A survey," IEEE Communications Surveys Tutorials, pp. 1-1, Mar 2019.

[18] Z. Liu, Z. Li, K. Wu et al., "Urban traffic prediction from mobility data using deep learning," IEEE Network, vol. 32, no. 4, pp. 40-46, July 2018.

[19] Y. Lv, Y. Duan, W. Kang et al., "Traffic flow prediction with big data: A deep learning approach," IEEE Trans. on Intelligent Transportation Systems, vol. 16, no. 2, pp. 865-873, Apr 2015.

[20] A. Dubey, N. Naik, D. Parikh et al., "Deep learning the city: Quantifying urban perception at a global scale," in Computer Vision - ECCV. Springer, 2016, pp. 196-212.

[21] K. D'Silva, A. Noulas, M. Musolesi et al., "Predicting the temporal activity patterns of new venues," EPJ Data Science, vol. 7, no. 1, p. 13, 2018.

[22] P. Vitello, A. Capponi, C. Fiandrino et al., "High-precision design of pedestrian mobility for smart city simulators," in Proc. IEEE ICC, 2018, pp. 1-6. 